\title{
ON THE HAUSDORFF DIMENSION OF NEWHOUSE PHENOMENA
}

\author{
PIERRE BERGER AND JACOPO DE SIMOI
}

\begin{abstract}
We show that at the vicinity of a generic dissipative homoclinic unfolding of a surface diffeomorphism, the Hausdorff dimension of the set of parameters for which the diffeomorphism admits infinitely many periodic sinks is at least $1 / 2$.
\end{abstract}

\section{INTRODUCTION}

The main goal of Palis program [Pal08] is to prove that given a generic, $d$ dimensional parameter family of $C^{r}$-diffeomorphisms of a compact manifold $(r \geq 1, d \geq 1)$, Lebesgue almost every parameter has the following property:

There exist finitely many invariant probabilities such that the union of their basins has full Lebesgue measure in the manifold.

According to the Palis program, homoclinic tangencies represent the main obstructions for a surface diffeomorphism to be uniformly hyperbolic. More precisely, Palis conjectured that every smooth surface diffeomorphism which is not uniformly hyperbolic can be perturbed to one having a hyperbolic periodic point $P$ whose stable and unstable manifolds have a quadratic tangency. This result has been proved in the $C^{1}$-topology by Pujals and Sambarino (see [PS00; see also [CP10] for a weaker $C^{1}$-version in higher dimension, and [MSS83, DL13] for a weaker version in complex dynamics). Homoclinic tangencies are associated to a remarkable dynamical feature discovered by Newhouse (see [New74, New79]) and which is nowadays referred to as the Newhouse phenomenon: in any neighborhood of a dissipative surface diffeomorphism exhibiting a non-degenerate homoclinic tangency, there exists a residual set of diffeomorphisms which admit infinitely many periodic sinks. Adapting some of Newhouse's results, Robinson (see [Rob83]) later proved that the phenomenon takes place for a residual set of parameters in a one-parameter family of diffeomorphisms which non-degenerately unfold an homoclinic tangency.

It is natural to ask whether this topologically significant behavior is also relevant from the point of view of probability (or more precisely, of prevalence). The first attempt towards a measure theoretic understanding of

Date: October 9, 2018.

$\mathrm{PB}$ is partially financed by the Balzan project of J. Palis and the Brazilian-French Network in Mathematics. JDS acknowledges partial NSERC support. 
Newhouse phenomena is due to Tedeschini-Lalli and Yorke (see [TLY86]): they considered a one-parameter unfolding of a homoclinic tangency involving a linear horseshoe, and showed that the set of parameters whose corresponding diffeomorphism admits infinitely many periodic simple sinks (i.e. sinks obtained with the Newhouse construction) is a null set for Lebesgue measure. In the same setting (one-parameter unfolding of an homoclinic tangency involving a linear horseshoe) Wang (see Wan90) proved that the Hausdorff dimension of the parameter set of diffeomorphisms admitting an infinite number of periodic simple sinks is strictly positive and smaller than $1 / 2$.

More recently, Gorodetski and Kaloshin (see [GK07]) obtained the measure-zero result in a much broader setting: they introduced a quantitative notion of combinatorial complexity of periodic orbit visiting a neighborhood of a homoclinic tangency, which they call cyclicity 1 . Their result shows that a prevalent dissipative surface diffeomorphism in a neighborhood of one exhibiting a non-degenerate homoclinic tangency has only finitely many sinks of cyclicity which is either bounded or negligible with respect to the period of the orbit.

The techniques of [New79, Rob83] do not apply to conservative surface diffeomorphisms; on the other hand, a clear analog of the Newhouse phenomenon still occurs in a vicinity of conservative diffeomorphisms exhibiting non-degenerate homoclinic tangencies, with elliptic islands filling in for the rôle of sinks. This result was finally established by Duarte and GonchenkoShilnikov (see [Dua99, GS03] and [Dua08] for the one-parameter version). In [DS13] the second author of this article proved an analog to TedeschiniLalli-Yorke and Wang result for the Standard Family of conservative diffeomorphisms in the large parameters regime: the set of (sufficiently large) parameters for which the Standard Family admits infinitely many simple sinks has zero Lebesgue measure and its Hausdorff dimension is not smaller than $1 / 4$.

In this paper we obtain a similar lower bound on the Hausdorff dimension for dissipative surface diffeomorphisms. We prove that the Newhouse parameter set for a generic family of sufficiently smooth diffeomorphisms (nondegenerately) unfolding a homoclinic tangency has Hausdorff dimension not smaller than $1 / 2$. It is important to stress that our lower bound takes into account non-simple sinks (and thus does not contradict Wang's result) and moreover does not assume linearity of the horseshoe. The proof of our result hinges on two crucial ingredients: the first one (Theorem 2.3) is an improved version of Newhouse construction of a wild hyperbolic set; the second one is Lemma 2.5 (proved in Ber14]), which provides precise estimates on the length of the stability range of a sink which is created by

\footnotetext{
${ }^{1}$ In their terminology, simple sinks which were considered above correspond to cyclicity one sinks.
} 
unfolding a homoclinic tangency via the Newhouse construction. As a consequence of these results, we obtain that the Hausdorff dimension of the simple Newhouse parameter set for strongly dissipative Hénon like families is close to one $1 / 2$ but not greater than $1 / 2$ (see Corollary 2.6). We conclude by using Palis-Takens renormalization (see Theorem 1.5).

The paper is organized as follows: in Section 1 we give a precise statement of our Main Theorem, and in Section 2 we collect the results used to give its proof; Appendix A provides a proof of Theorem 2.3 based on renormalization of Hénon-like maps.

\section{Statement of the Main Theorem}

1.1. Main definitions. Let $f$ be a $C^{r}$-diffeomorphism of a surface $M, r \geq$ 1. A point $p \in M$ is a periodic sink if $p$ is periodic, $f^{n}(p)=p$, and all eigenvalues of the differential $D_{p} f^{n}$ have modulus less than 1 . A point $p \in M$ is a saddle periodic point if $D_{p} f^{n}$ has one eigenvalue of modulus less than 1 the another one of modulus greater than 1 . The local stable and unstable manifolds of $p$ are respectively:

$$
\begin{aligned}
& W_{\epsilon}^{s}(p ; f):=\left\{y \in M: \epsilon>d\left(f^{n}(p), f^{n}(y)\right) \rightarrow 0,0 \leq n \rightarrow+\infty\right\}, \\
& W_{\epsilon}^{u}(p ; f):=\left\{y \in M: \epsilon>d\left(f^{n}(p), f^{n}(y)\right) \rightarrow 0,0 \geq n \rightarrow-\infty\right\} .
\end{aligned}
$$

By Hadamard-Perron Theorem, for sufficiently small $\epsilon$, they are embedded $C^{r}$-curves; on the other hand the stable and unstable manifolds of $p$

$$
W^{s}(p ; f):=\bigcup_{n \leq 0} f^{n}\left(W_{\epsilon}^{s}(p ; f)\right) \quad W^{u}(p ; f):=\bigcup_{n \geq 0} f^{n}\left(W_{\epsilon}^{u}(p ; f)\right)
$$

are immersed submanifolds. We say that a saddle point $p$ has a homoclinic tangency if these two immersed submanifolds are tangent at a point.

A family $\left(f_{a}\right)_{a \in \mathbb{R}}$ of diffeomorphisms of a surface $M$ is of class $C^{r}$ if the $\operatorname{map} \mathbb{R} \times M \ni(a, z) \mapsto f_{a}(z) \in M$ is of class $C^{r}$. It is well known that, if $f_{0}$ has a saddle fixed point $\Omega_{0}$, then this point persists as a saddle fixed point $\Omega_{a}$ of $f_{a}$ for $a$ small. Hence there exists a $C^{r}$-chart $\phi_{a}$ of a neighborhood $D$ of $\Omega_{a}$ which maps $\Omega_{a}$ to $0, W_{\epsilon}^{s}\left(\Omega_{a} ; f_{a}\right)$ onto $\{0\} \times(-1,1)$ and $W_{\epsilon}^{u}\left(\Omega_{a} ; f_{a}\right)$ onto $(-1,1) \times\{0\}$. By [HPS77], the following map can be chosen to be $C^{r}$ :

$$
\phi:(-\eta, \eta) \times D \ni(a, z) \mapsto\left(a, \phi_{a}(z)\right) \in(-\eta, \eta) \times \mathbb{R}^{2} .
$$

We say that the family $\left(f_{a}\right)_{a}$ nondegenerately unfolds a homoclinic tangency at $a_{0}$ of the periodic point $\Omega$, if there exist $P=(p, 0) \in D$ sent by $f_{a_{0}}^{N}$ to a point $Q=(0, q) \in D$, and a neighborhood $D_{P} \ni P$, such that, for every $a$ sufficiently small, $f_{a}^{N} D_{P} \subset D$, and $f_{a}^{N} \mid D_{P}$ has the form:

$$
P+(x, y) \in D_{P} \mapsto Q+\left(\xi x^{2}+a+\gamma \cdot y, \zeta \cdot x\right)+E_{a}(x, y) \in D
$$

where $\zeta \in \mathbb{R}, \xi, \gamma$ are non-zero constants (all independent of $a$ ) and $E_{a}=$ $\left(E_{a}^{1}, E_{a}^{2}\right) \in C^{r}\left(\mathbb{R} \times \mathbb{R}^{2}, \mathbb{R}^{2}\right)$ satisfies at $(x, y)=0$ and $a=0$ :

$$
\left\{\begin{array}{l}
E_{a}^{1}=\partial_{x} E_{a}^{1}=\partial_{y} E_{a}^{1}=\partial_{a} E_{a}^{1}=\partial_{x x} E_{a}^{1}=0 \\
E_{a}^{2}=\partial_{x} E_{a}^{2}=0
\end{array}\right.
$$


Note that non-degenerate homoclinic unfolding tangency are stable by $C^{2}$ perturbations, and open and dense among families having a homoclinic tangency at some parameter. Let us illustrate this fact on a crucial class of maps of $\mathbb{R}^{2}$.

Definition 1.1 (Hénon like maps). A Hénon map is a map of $\mathbb{R}^{2}$ of the following form, with $a, b \in \mathbb{R}$ :

$$
h_{a b}: \mathbb{R}^{2} \ni(x, y) \mapsto\left(x^{2}+a+y, b x\right) \in \mathbb{R}^{2} .
$$

A Hénon $C^{r}$-like map is a map $f$ of the form

$$
f_{a}: \mathbb{R}^{2} \ni(x, y) \mapsto\left(x^{2}+a+y, b x\right)+\left(A_{a}(x, y), b \cdot B_{a}(x, y)\right) \in \mathbb{R}^{2} .
$$

where $A_{a}$ and $B_{a}$ are $C^{r}$-small maps. The map $f_{a}$ is Hénon $C^{r}$ - $\delta$-like if both $A_{a}$ and $B_{a}$ have $C^{r}$-norm at most $\delta$.

If $A_{a}$ and $B_{a}$ depend on the parameter $a \in(-3,1)$ so that $(x, y, a) \mapsto$ $A_{a}(x, y)$ and $(x, y, a) \mapsto B_{a}(x, y)$ are of class $C^{r}$ with norms both smaller than $\delta$, then the family $\left(f_{a}\right)_{a}$ is Hénon $C^{r}-\delta$-like.

Example 1.2 (Unfolding of homoclinic tangencies in Hénon-like families). The family $\left(h_{a 0}\right)_{a}$ nondegenerately unfolds a homoclinic tangency at $a_{0}=$ -2 of the fixed point $\Omega$, with $\Omega_{a_{0}}:=(2,0)$. Hence for $b$ small and $\delta$ small, any Hénon $C^{2}-\delta$-like family $\left(f_{a}\right)_{a}$ nondegenerately unfolds a homoclinic tangency at a certain $a \approx a_{0}$ of the hyperbolic continuation of $\Omega$.

1.2. Main results. Here is the main result of this note:

Main Theorem. Let $r$ be sufficiently large (e.g. $r \geq 24)$ and let $\left(f_{\mu}\right)_{\mu \in \mathbb{R}}$ be a generic family of $C^{r}$ surface diffeomorphisms (nondegenerately) unfolding a homoclinic tangency at $\mu_{0}$ of a periodic point $\Omega$. If $\left|\operatorname{det}\left(D_{\Omega_{\mu_{0}}} f_{\mu_{0}}\right)\right|<1$, then the following set has Hausdorff dimension at least 1/2:

$$
\mathcal{N}:=\left\{\mu \in \mathbb{R}: f_{\mu} \text { has infinitely many sinks }\right\} .
$$

Remark 1.3. In the Main Theorem, we need the family to be sufficiently smooth and to satisfy some genericity conditions to satisfy the hypotheses of a version of Sternberg linearization theorem due to Sell (see [Sel85]). Even if it seems to be possible to weaken the assumptions (and prove the result for e.g. $C^{3}$-families not necessarily generic), we do not pursue this task here.

To show the main Theorem, we will prove the following result:

Proposition 1.4. For every Hénon $C^{4}$-like family $\left(f_{a}\right)_{a}$, with small determinant, the following set has Hausdorff dimension at least $1 / 2$ :

$$
\mathcal{N}:=\left\{a \in \mathbb{R}: f_{a} \text { has infinitely many sinks }\right\} .
$$

Proposition 1.4 implies the Main Theorem, by the following result; for the proof see ([Ber14, Theorem 1.4 and Remark 1.5]) 
Theorem 1.5 (Palis-Takens revisited). For any $\delta>0$ and any one-parameter family $\left(f_{\mu}\right)_{\mu}$ as in the Main Theorem, there exists, for each positive integer $n$, a reparametrization $\mu=M_{n}(\tilde{\mu})$ of the $\mu$ variable and $\tilde{\mu}$-dependent coordinate transformations $(\tilde{x}, \tilde{y}) \mapsto(x, y)=\Psi_{n, \tilde{\mu}}(\tilde{x}, \tilde{y})$ such that:

- for each compact set $K$ in the $(\tilde{x}, \tilde{y}, \tilde{\mu})$-space, the images of $K$ under the maps

$$
(\tilde{x}, \tilde{y}, \tilde{\mu}) \mapsto(x, y, \mu)=\left(\Psi_{n, \tilde{\mu}}(\tilde{x}, \tilde{y}), M_{n}(\tilde{\mu})\right)
$$

converge, for $n \rightarrow \infty$, in the $(x, y, \mu)$ space to $\left(P, \mu_{0}\right)$;

- the domains of the maps

$$
\begin{aligned}
& (\tilde{x}, \tilde{y}, \tilde{\mu}) \mapsto \mathcal{R} f_{\tilde{\mu}}(\tilde{x}, \tilde{y}):=\Psi_{n, \tilde{\mu}}^{-1} \circ\left((f \mid D)^{n} \circ\left(f^{N} \mid D_{P}\right)\right)_{M_{n}(\tilde{\mu})} \circ \Psi_{n, \tilde{\mu}}(\tilde{x}, \tilde{y}) \\
& \text { converge, for } n \rightarrow \infty \text {, to all } \mathbb{R}^{3} \text {; } \\
& \text { - when } n \text { is sufficiently large, the family }\left(\mathcal{R} f_{\tilde{\mu}}\right)_{\tilde{\mu}} \text { is Hénon } C^{4}-\delta \text {-like } \\
& \text { and with determinant smaller than } \delta .
\end{aligned}
$$

\section{Proof of Proposition 1.4}

The proof is based on the Newhouse construction of a wild hyperbolic set and fine estimates in parameter space on the distribution of parameters with many attracting periodic points.

2.1. Wild hyperbolic sets. We recall that an invariant compact set $K \subset$ $M$ is hyperbolic for a diffeomorphism $f$ of $M$ if the restriction of the tangent bundle $T M$ to $K$ splits into two $D f$-invariant directions $E^{s}$ and $E^{u}$ which are contracted, respectively, by $D f$ and $D f^{-1}$.

The set $K$ is basic if, moreover, it is transitive and the closure of the set of periodic points of $f$ contains $K$. This enables us to define, for $\epsilon>0$, as we did for periodic points, the $\epsilon$-local stable and unstable manifolds $W_{\epsilon}^{s}(z)$ and $W_{\epsilon}^{u}(z)$ of points $z \in K$; let $W_{\epsilon}^{s}(K)=\bigcup_{z \in K} W_{\epsilon}^{s}(z)$ and $W_{\epsilon}^{u}(K)=$ $\bigcup_{z \in K} W_{\epsilon}^{u}(z)$

For every $f^{\prime} C^{1}$-close to $f$, there exists (see [Yoc95]), a (continuous) embedding $i_{f^{\prime}}$

$$
i_{f^{\prime}}: K \hookrightarrow \mathbb{R}^{2}
$$

so that $i_{f}$ is the canonical injection and $f^{\prime} \circ i_{f^{\prime}}=i_{f^{\prime}} \circ f \mid K$. The map $f^{\prime} \mapsto i_{f^{\prime}}$ is smooth from a $C^{1}$-neighborhood of $f$ into the space $C^{0}\left(K, \mathbb{R}^{2}\right)$. Let us denote for $f^{\prime} C^{1}$-close to $f$ and $\Omega \in K$ :

$$
K\left(f^{\prime}\right):=i_{f^{\prime}}(K) \quad \Omega\left(f^{\prime}\right):=i_{f^{\prime}}(\Omega) .
$$

$K\left(f^{\prime}\right)$ (resp. $\left.\Omega\left(f^{\prime}\right)\right)$ is called the hyperbolic continuation of $K$ (resp. $\Omega$ ). In particular $K(f)=K$.

Definition 2.1. We say that a basic set $K$ is wild, if there exists $L>0$ such that, for every $C^{2}$-perturbation $f^{\prime}$ of $f$, there exists $\Omega_{f^{\prime}} \in K$ such that $W_{L}^{s}\left(\Omega_{f^{\prime}}\left(f^{\prime}\right), f^{\prime}\right)$ and $W_{L}^{u}\left(\Omega_{f^{\prime}}\left(f^{\prime}\right), f^{\prime}\right)$ have a quadratic tangency. 
Remark 2.2. Even if $W_{L}^{s}\left(\Omega_{f}(f), f\right)$ and $W_{L}^{u}\left(\Omega_{f}(f), f\right)$ have a quadratic tangency, there exists $f^{\prime}$ arbitrarily close to $f$ so that $W_{L}^{s}\left(\Omega_{f}\left(f^{\prime}\right), f^{\prime}\right)$ and $W_{L}^{u}\left(\Omega_{f}\left(f^{\prime}\right), f^{\prime}\right)$ are not tangent. Hence $\Omega_{f}\left(f^{\prime}\right)$ is in general not equal to $\Omega_{f^{\prime}}\left(f^{\prime}\right)$. Since $K$ is totally disconnected, the map $f^{\prime} \mapsto \Omega_{f^{\prime}} \in K$ is not continuous; neither is the map $f^{\prime} \mapsto \Omega_{f^{\prime}}\left(f^{\prime}\right) \in K\left(f^{\prime}\right)$.

By Example 1.2, the family of maps $\left(f_{a}\right)_{a}$ nondegenerately unfolds a homoclinic tangency of a fixed point $\Omega$ with small determinant. By [New79, Theorem 3], there exists $a_{0} \approx-2$ such that $f_{a_{0}}$ leaves invariant a wild basic set $\tilde{K}$. Actually, we shall give a modern proof of this result in order to bound the expansion of the unstable direction uniformly on the determinant of the Hénon-like map.

In the case of family of diffeomorphisms $\left(f_{a}\right)_{a}$, to make the notation less cumbersome, $K(a)$ and $\Omega(a)$ stand for the hyperbolic continuations $K\left(f_{a}\right)$ and $\Omega\left(f_{a}\right)$ respectively.

Theorem 2.3 (Newhouse Theorem revisited). There exists $\Lambda>1$ such that for any Hénon $C^{4}$-like family $\left(f_{a}\right)_{a}$ with small determinant, there exist $a_{0} \approx-2, L>0$, an open parameter interval $I \ni a_{0}$ and a basic set $\tilde{K}\left(a_{0}\right)$, such that for any $a_{1} \in I$, the following properties hold:

(i) there exists a Riemannian metric for which the expansion of $\tilde{K}\left(a_{1}\right)$ in the unstable direction is at least $\Lambda$;

(ii) there exists $\Omega_{a_{1}}\left(a_{0}\right) \in \tilde{K}\left(a_{0}\right)$ close to $\Omega\left(a_{0}\right)$ so that $W_{L}^{s}\left(\Omega_{a_{1}}(a), f_{a}\right)$ is tangent to $W_{L}^{u}\left(\Omega_{a_{1}}(a), f_{a}\right)$ at $a=a_{1}$, via a quadratic tangency, that $\left(f_{a}\right)_{a}$ nondegenerately unfolds.

In particular, $\tilde{K}\left(a_{0}\right)$ is wild.

Remark 2.4. Item (i) in the above theorem is not proved in the versions of the Newhouse Theorem currently available in the literature. It will be important that $\Lambda>1$ does not depend on the (possibly very small) determinant of $f_{a}$.

In Appendix A we give a proof of Theorem 2.3 based not only on Newhouse thickness but also on renormalization techniques.

2.2. Parameters with many attracting periodic points. In order to get refined estimates on the distribution of parameters with many attracting periodic points, we will use the following:

Lemma 2.5 ([Ber14, Lemma 3.2]). Let $\left(f_{a}\right)_{a}$ be a Hénon $C^{2}$-like family, of class $C^{4}$, such that a non-degenerate homoclinic unfolding holds with a periodic point $\Omega$ in a hyperbolic set $K$ at $a=a_{0}$.

Then there exist $C>0, M \in \mathbb{N}$ and $N \geq 0$ such that for every periodic point $\Omega^{\prime}$ of period $p^{\prime}$ close to $\Omega$, a non-degenerate homoclinic unfolding holds with $\Omega^{\prime}$ at $a_{0}^{\prime}$ close to $a_{0}$.

Moreover if $p^{\prime} \geq M$, by denoting by $\sigma$ the unstable eigenvalue of $D_{\Omega^{\prime}} f_{a_{0}^{\prime}}^{p^{\prime}}$, there exists a parameter interval $I_{\Omega^{\prime}}$ such that: 
- for every $a \in I_{\Omega^{\prime}}$, the map $f_{a}$ has an attracting cycle of minimal period $p^{\prime}+N$,

- the length of $I_{\Omega^{\prime}}$ is in $\left[\sigma^{-2} / C, C \sigma^{-2}\right]$,

- the distance between $I_{\Omega^{\prime}}$ and $a_{0}^{\prime}$ is in $\left[\sigma^{-1} / C, C \sigma^{-1}\right]$.

The above lemma provides, in our general setting, the parameter space estimate which has been essential in most previous results; for instance, in TLY86, Wan90] this estimate follows from the linearity of the horseshoe; in our case it is obtained via renormalization techniques. We can employ it, en passant, to recover a version of Tedeschini-Lalli-Yorke and Wang results applicable to our broader setting.

Corollary 2.6. Given a horseshoe $K$ for a Hénon $C^{4}$-like family, and a nondegenerate unfolding of a homoclinic tangency, the set $\mathcal{N}_{1}(K)$ of parameters with infinitely many attracting simpl $\left.\right|^{2}$ sinks has Hausdorff dimension at most $1 / 2$. In particular, it is a null set for Lebesgue measure.

Proof. For $\epsilon>0$ small and $i \in \mathbb{N}$, define the intervals $I_{i}:=[i \epsilon,(i+1) \epsilon)$. Let $\operatorname{Per}_{i}$ be the set of periodic orbits of $K$ whose mean expansion $\sigma \in I_{i}$ and let $K_{i}$ be the closure of $\operatorname{Per}_{i}$; clearly $K_{i}$ is a (hyperbolic) basic set. We claim that $\mathcal{N}_{1}(K)$ is the union $\mathcal{N}_{1}(K)=\bigcup_{i} \mathcal{N}_{1}\left(K_{i}\right)$ : in fact every $f \in \mathcal{N}_{1}(K)$ has infinitely many simple sinks. Each such sink is 1-1 associated with an integer $n$ and a fixed point of $f^{n}$. By compactness, only finitely many $K_{i}$ are nonempty, thus for every $f \in \mathcal{N}_{1}(K)$, there exists $i$ so that there are infinitely many sinks associated to periodic saddles in $K_{i}$, which implies that $f \in \mathcal{N}_{1}\left(K_{i}\right)$.

Let $h_{i}$ be the topological entropy of $K_{i}$, by Ruelle inequality it holds

$$
h_{i} \leq \sup \log \sigma \leq \log ((i+1) \epsilon) .
$$

On the other hand, the number of points in $\operatorname{Per}_{i}$ fixed by $f^{n}$ is at most $\exp \left(n h_{i}\right) \leq((i+1) \epsilon)^{n}$. Given $p \in \operatorname{Fix} f^{n} \mid K_{i}$ of period $n$, Lemma2.5 implies that the attracting cycle associated to it exists for an interval of parameters $I_{p}$ of length Const $\sigma^{-2 n} \leq \operatorname{Const}(i \epsilon)^{-2 n}$.

The family $\left(I_{p}\right)_{p \in \operatorname{Fix} f^{n}, n \geq N}$ is a covering of $\mathcal{N}_{1}\left(K_{i}\right)$ for every $N$. We notice that

$$
\sum_{p \in \operatorname{Fix} f^{n}, n \geq N}\left|I_{p}\right|^{s} \leq \text { Const } \sum_{n \geq N}(i \epsilon)^{-2 n s}((i+1) \epsilon)^{n} .
$$

The above series converges if $(i \epsilon)^{-2 s}(i+1) \epsilon<1$, thus the Hausdorff dimension of $\mathcal{N}_{1}\left(K_{i}\right)$ is at most $s$. The above condition on $s$ can be made arbitrarily close to $s=1 / 2$ by taking $\epsilon$ small.

Let us go back to the proof of our main result. We recall that, by Theorem 2.3. for every $a_{1} \in I \ni a_{0}$, the map $f_{a_{1}}$ has a wild basic set $\tilde{K}\left(a_{1}\right)$,

\footnotetext{
${ }^{2}$ For our purposes we can define a cycle to be simple (with respect to a given homoclinic tangency) if it appears as a consequence of the Newhouse construction, of which Lemma 2.5 is a refinement. In particular, there exist a neighborhood of the tangency point which contains only one element of every simple cycle.
} 
which is the hyperbolic continuation of $\tilde{K}\left(a_{0}\right)$. Also, there exists $L$, such that for every $a_{1} \in I$, there exists $\Omega_{a_{1}}\left(a_{1}\right) \in \tilde{K}\left(a_{1}\right)$ close to $\Omega\left(a_{1}\right)$ so that $W_{L}^{s}\left(\Omega_{a_{1}}\left(a_{1}\right), f_{a_{1}}\right)$ is tangent to $W_{L}^{u}\left(\Omega_{a_{1}}\left(a_{1}\right), f_{a_{1}}\right)$, via a quadratic tangency, unfolded nondegenerately by $\left(f_{a}\right)_{a}$.

Since periodic points are dense in $\tilde{K}$, there exists, for any $a_{1}$, a periodic point $\Omega_{a_{1}}^{\prime}\left(a_{1}\right)$ nearby $\Omega_{a_{1}}\left(a_{1}\right)$ and a parameter $a_{1}^{\prime}$ nearby $a_{1}$, so that $W_{L}^{s}\left(\Omega_{a_{1}}^{\prime}\left(a_{1}^{\prime}\right), f_{a_{1}^{\prime}}\right)$ and $W_{L}^{u}\left(\Omega_{a_{1}}^{\prime}\left(a_{1}^{\prime}\right), f_{a_{1}^{\prime}}\right)$ have a quadratic tangency, unfolded nondegenerately by $\left(f_{a}\right)_{a}$.

We will bound the distance between $a_{1}^{\prime}$ and $a_{1}$ as a function of the unstable eigenvalue of $D_{\Omega_{a_{1}}^{\prime}\left(a_{1}^{\prime}\right)} f_{a_{1}^{\prime}}^{p}$, with $p$ being the period of $\Omega_{a_{1}}^{\prime}$, in view of Theorem 2.3. Since the unfolding is nondegenerate, the distance between $a_{1}^{\prime}$ and $a_{1}$ is of the same order as the distance between $\Omega_{a_{1}}\left(a_{1}\right)$ and $\Omega_{a_{1}}^{\prime}\left(a_{1}\right)$. Furthermore, by a standard computation, there exists $C>0$, such that for all such $a_{1}, a_{1}^{\prime}, \Omega_{a_{1}}, \Omega_{a_{1}}^{\prime}$, and $n \geq 0$ we have:

$$
\begin{array}{r}
\frac{d\left(a_{1}, a_{1}^{\prime}\right)}{C}<d\left(\Omega_{a_{1}}\left(a_{1}\right), \Omega_{a_{1}}^{\prime}\left(a_{1}\right)\right)<C d\left(a_{1}, a_{1}^{\prime}\right) \\
\frac{n}{C}<\frac{\partial_{a}\left\|D_{\Omega_{a_{1}}^{\prime}} f_{a}^{n}\right\|}{\left\|D_{\Omega_{a_{1}}^{\prime}} f_{a}^{n}\right\|}<C n, \quad \forall a \in I .
\end{array}
$$

By the construction of a Markov partition by Bowen [Bow70, there exist $N \geq 1$, a matrix $A \in M_{n}(\{0,1\})$ such that $\tilde{K}$ is homeomorphic to $\Sigma_{A}:=$ $\left\{\left(j_{i}\right)_{i} \in\{1, \ldots, N\}^{\mathbb{Z}}: A_{j_{i} j_{i+1}}=1, \forall i \in \mathbb{Z}\right\}$, via a homeomorphism $h$ which conjugates $f \mid \tilde{K}$ to the shift $\sigma: \Sigma_{A} \ni\left(j_{i}\right)_{i} \mapsto\left(j_{i+1}\right)_{i} \in \Sigma_{A}$.

Moreover, given any $\epsilon>0$, we can find $h, N$ and $A$ so that, for every $j \in\{1, \ldots, N\}$, the set $R_{j} \subset \tilde{K}$ of points $z$ such that the 0 -coordinate of $h(z)$ is equal to $j$ satisfies that the diameter of $R_{j}$ is less than $\epsilon$. We notice that $R_{j}$ is a clopen set of $\tilde{K}$.

An admissible chain from $i$ to $j$ of length $m$ is a sequence $i=j_{1}, \ldots, j_{m}=$ $j$, such that $A_{j_{k} j_{k+1}}=1$ for every $k$.

Fact 2.7. By transitivity of $\tilde{K}$, there exists $M_{T} \geq 0$ such that for all $i, j \in$ $\{1, \cdots, N\}$, there exists an admissible chain from $i$ to $j$ of length $m \leq M_{T}$.

For $n<m \in \mathbb{Z}$, an admissible chain $\underline{j}:=\left(j_{i}\right)_{i=n}^{m} \in\{1, \ldots, N\}^{m-n+1}$, define the sets:

$$
R_{\underline{j}}:=\bigcap_{i=n}^{m} f^{i}\left(R_{j_{i}}\right)
$$

Let $z \in \tilde{K}$ and set for $n \geq 0$ (we omit the obvious dependence on $a$ ):

$$
\begin{aligned}
\lambda_{n}(z) & :=\left\|D_{z} f^{n} \mid E^{s}\right\| & \sigma_{n}(z) & :=\left\|D_{z} f^{n} \mid E^{u}\right\|, \\
\lambda_{-n}(z) & :=\left\|D_{f^{-n}(z)} f^{n} \mid E^{s}\right\| & \sigma_{-n}(z) & :=\left\|D_{f^{-n}(z)} f^{n} \mid E^{u}\right\| .
\end{aligned}
$$

A classical argument of binding proves the following 
Lemma 2.8. There exists $C>0$, such that for all $m \leq 0 \leq n$, every admissible chain $\underline{j}:=\left(j_{i}\right)_{i=m}^{n}$, and all $z, z^{\prime} \in R_{\underline{j}}$, we have

$$
\begin{gathered}
\lambda_{n}(z) \leq C \lambda_{n}\left(z^{\prime}\right) \quad \text { and } \quad \sigma_{n}(z) \leq C \sigma_{n}\left(z^{\prime}\right) . \\
\lambda_{m}(z) \leq C \lambda_{m}\left(z^{\prime}\right) \quad \text { and } \quad \sigma_{m}(z) \leq C \sigma_{m}\left(z^{\prime}\right) .
\end{gathered}
$$

Corollary 2.9. There exists $C>0$, such that such that for all $m \leq$ $0 \leq n$, any admissible chain $\underline{j}:=\left(j_{i}\right)_{i=n}^{m}$, the diameter of $R_{j}$ is less than $C \max \left(\sigma_{n}^{-1}(z), \lambda_{m}(z)\right)$ for any $z \in R_{\underline{j}}$.

Proposition 2.10. There exists $C>0$, such that, for any $a_{1} \in I$ and $n \geq 0$, with $n^{\prime}=n^{\prime}(n)$ such that

$$
\lambda_{-n^{\prime}-1}\left(\Omega_{a_{1}}\left(a_{1}\right)\right) \leq \sigma_{n}^{-1}\left(\Omega_{a_{1}}\left(a_{1}\right)\right) \leq \lambda_{-n^{\prime}}\left(\Omega_{a_{1}}\left(a_{1}\right)\right),
$$

let $\underline{j}:=\left(j_{i}\right)_{i=-n^{\prime}}^{n} \in\{1, \ldots, N\}^{n+n^{\prime}+1}$, such that $\Omega_{a_{1}}$ belongs to $R_{j}$. Then at $a=a_{1}$ and $z=\Omega_{a_{1}}$

$$
\left(\sigma_{n} \sigma_{-n^{\prime}}\right) \leq C\left(\sigma_{n}\right)^{1-C / \log b}
$$

Proof. For ease of exposition, although with abuse of notation, let us denote $\lambda_{-n^{\prime}}\left(\Omega_{a_{1}}\left(a_{1}\right)\right)$ by $\lambda_{-n^{\prime}}, \sigma_{-n^{\prime}}\left(\Omega_{a_{1}}\left(a_{1}\right)\right)$ by $\sigma_{-n^{\prime}}$, and so on. However we keep in mind the dependence in $a_{1}$ and $z$.

Since the determinant of $f$ is smaller than $b$, there exists $C>0$ such that for all $a_{1} \in I$ and $n \in \mathbb{Z}$ :

$$
\sigma_{n} \lambda_{n} \leq C b^{|n|}
$$

Moreover, since the norm of $D f$ is bounded by 5 on the complement of the basin of infinity, and by $(i)$ of Theorem 2.3 , there exists $C>0$ such that for all $a_{1} \in I$ and $n \in \mathbb{Z}$ :

$$
5^{|n|} \geq \sigma_{n} \geq C \Lambda^{|n|} .
$$

Hence there exists $C>0$ such that for all $a_{1} \in I$ and $n \geq 0$ :

$$
\lambda_{-n^{\prime}} \leq C(b / \Lambda)^{n^{\prime}} \leq C b^{n^{\prime}} .
$$

But $5^{-n} \leq \sigma_{n}^{-1} \leq \lambda_{-n^{\prime}}$. Thus

$$
5^{-n} \leq C b^{n^{\prime}} \Rightarrow n^{\prime} \leq \frac{n \log 5+\log C}{-\log b} .
$$

Consequently:

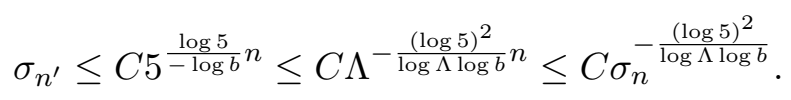

By Corollary 2.9, if $\Omega_{a_{1}}$ belongs to $R_{j}$, with $\underline{j}(n):=\left(j_{i}\right)_{i=-n^{\prime}}^{n}$, then the diameter of $R_{j(n)}$ is less than $C \sigma_{n}^{-1}$.

By Fact 2.7. there exists a periodic point $\Omega_{a_{1}}^{(n)}$ in $R_{\underline{j}(n)}$ of period $p(n) \in$ $\left[n+n^{\prime}, n+n^{\prime}+M_{T}\right]$. By (1) and Lemma 2.5 we know that $f_{a}$ will nondegenerately unfold a homoclinic tangency for $\Omega_{a_{1}}^{(n)}(a)$ at $a=a_{1}^{(n)}$ that is 
$C \sigma_{n}^{-1}$-close to $a_{1}$. Indeed:

$$
\begin{gathered}
d\left(a_{1}, a_{1}^{(n)}\right) \leq C d\left(\Omega_{a_{1}}\left(a_{1}\right), \Omega_{a_{1}}^{(n)}\left(a_{1}\right)\right) \leq \sup _{a \in\left[a_{1}, a_{1}^{(n)}\right]} \frac{C}{\sigma_{-n}(a)} \\
\leq \frac{C}{\sigma_{-n}\left(a_{1}\right)}+C d\left(a_{1}, a_{1}^{(n)}\right) \sup _{\left[a_{1}, a_{1}^{(n)}\right]} \partial_{a} \frac{1}{\sigma_{-n}(a)}=\frac{C}{\sigma_{-n}\left(a_{1}\right)}+o\left(d\left(a_{1}, a_{1}^{(n)}\right)\right)
\end{gathered}
$$

Moreover, again by Lemma 2.5, if $n$ is large enough (e.g. $n \geq M$ certainly suffices), there exists an interval of parameters $I_{n}$, at a distance dominated by $\sigma_{n}^{-1}$ of $a_{1}^{(n)}$ (and consequently of $a_{1}$ ), which is of length proportional to $\left(\sigma_{-n^{\prime}} \sigma_{n}\right)^{-2} \geq C\left(\sigma_{n}\right)^{-2+\frac{C}{\log (b)}}$, such that for every $a \in I_{n}$, the map $f_{a}$ has an attracting periodic point of minimal period $p(n)+N$. Let us summarize the content of this subsection in the following

Proposition 2.11. There exist $\Lambda^{\prime}>\Lambda>1, C, D>0$, an open interval Iof parameters $a$, such that for every $a_{1} \in I$, there exist a sequence of intervals $\left(I_{k}\left(a_{1}\right)\right)_{k}$ and a sequence of positive integers $\left(m_{k}\right)_{k}$ such that:

- $I_{k}\left(a_{1}\right)$ is at a distance at most $D\left|I_{k}\right|^{1 / 2-C / \log b}$ of $a_{1}$,

- there exists $\bar{M}>0$ so that $m_{k+\bar{M}}>m_{j}$ for any $0<j<k$;

- $f_{a}$ has an attracting periodic point $p_{k}(a)$ of period $m_{k}$, for every $a \in I_{k}\left(a_{1}\right)$;

- $\Lambda\left|I_{k+1}\left(a_{1}\right)\right|<\left|I_{k}\left(a_{1}\right)\right|<\Lambda^{\prime}\left|I_{k+1}\left(a_{1}\right)\right|$.

2.3. Lower bound on the Hausdorff dimension of $\mathcal{N}$. Proposition 2.11 enables us to build subsets of $\mathcal{N}$ which are particularly well-suited to obtain bounds on the Hausdorff dimension. Let $n_{l} \rightarrow \infty$ be a sequence of natural numbers which diverges super-exponentially fast; to fix ideas, take $n_{l}=2^{C \cdot 2^{l}}$ where $C>1$ is a large constant. We will now construct a Cantor set by the following inductive procedure. Let $\mathcal{I}_{0}=I$ be the interval given by Proposition 2.11. Then, for $l>0$, assume by induction that we defined sets $\mathcal{I}_{0} \supset \cdots \supset \mathcal{I}_{l-1}$ satisfying the following properties:

- for any $a \in \mathcal{I}_{l-1}, f_{a}$ has at least $l-1$ attracting periodic points of period at most $m_{n_{l-1}+\bar{M}}$;

- for $0 \leq k \leq l-1, \mathcal{I}_{k}$ is a finite disjoint union $\mathcal{I}_{k}=\bigsqcup_{j} \mathcal{I}_{k}^{j}$ where $\mathcal{I}_{k}^{j}$ are intervals such that

$$
C^{\prime-1} \Lambda^{-n_{k}} \leq\left|\mathcal{I}_{k}^{j}\right| \leq C^{\prime} \Lambda^{-n_{k}}, \quad C^{\prime}:=\sqrt{\Lambda^{\prime}}
$$

moreover, the intervals $\mathcal{I}_{k}^{j}$ are separated by gaps of at least $\epsilon_{k}=$ $D^{\prime} \Lambda^{-\alpha n_{k}}$, for some $D^{\prime}>0$ and $\alpha=(1 / 2-C / \log b)$;

- for $0<k \leq l-1$, each interval $\mathcal{I}_{k-1}^{j}$ contains a number $\kappa_{k}^{j}$ of components of $\mathcal{I}_{k}$ so that, for some $C^{\prime \prime}>0$

$$
\kappa_{k}^{j} \geq C^{\prime \prime} \Lambda^{-n_{k-1}+\alpha n_{k}} .
$$


We then define the set $\mathcal{I}_{l}$ as follows: partition each interval $\mathcal{I}_{l-1}^{j}$ in subintervals of length ranging in $\left(2 D^{\prime} \Lambda^{-\alpha n_{l}}, 4 D^{\prime} \Lambda^{-\alpha n_{l}}\right)$, with $D^{\prime}$ fixed to be specified later. For any parameter $a$ that is an interna $]^{3}$ endpoint of this subdivision, we apply Proposition 2.11: we choose $k$ (depending on $a$ ) so that

$$
C^{\prime-1} \Lambda^{-n_{l}} \leq\left|I_{k}(a)\right| \leq C^{\prime} \Lambda^{-n_{l}}
$$

By design, for all $a^{\prime} \in I_{k}(a), f_{a^{\prime}}$ admits an attracting periodic orbit of period $m_{k}$; moreover, our construction ensures that $\rho n_{l} \leq k+k_{0} \leq n_{l}$ with $\rho=\log \Lambda / \log \Lambda^{\prime}$ and $k_{0}$ a constant independent of $k, l$; if we choose $C$ in the definition of $n_{l}$ to be large enough, we can guarantee that $m_{k}>m_{n_{l-1}+\bar{M}}$, which ensures that the periodic orbit we found at this step is distinct from the ones obtained at any of the previous steps.

Moreover, we know that the distance of $I_{k}(a)$ from $a$ is at most

$$
D C^{\prime \alpha} \Lambda^{-\alpha n_{l}}
$$

therefore, if we let $D^{\prime}=4 D C^{\alpha}$, such an interval will be separated from the one which could be obtained applying this construction to any other internal endpoint of our subdivision by at least $D^{\prime} \Lambda^{-\alpha n_{l}}$. We repeat this construction for all internal endpoints and we define $\mathcal{I}_{l}$ to be the disjoint union of all such intervals. Observe that, by definition, each interval $\mathcal{I}_{l-1}^{j}$ contains a number $\kappa_{l}^{j}$ of intervals of $\mathcal{I}_{l}$ which can be bounded as follows:

$$
\kappa_{l}^{j} \geq C^{\prime \prime} \Lambda^{-n_{l-1}+\alpha n_{l}}
$$

with $C^{\prime \prime}=\left(6 C^{\prime} D^{\prime}\right)^{-1}$. This concludes the proof of our induction step.

We can choose $C$ sufficiently large in the definition of the sequence $n_{l}$ to ensure that $\kappa_{l}^{j} \geq 2$ for any $l$ and $j$. Let $\mathcal{I}=\bigcap_{l=0}^{\infty} \mathcal{I}_{l}$; then by construction, for any $a \in \mathcal{I}, f_{a}$ admits infinitely many sinks, i.e. $\mathcal{I} \subset \mathcal{N}$. In order to obtain a lower bound on the Hausdorff dimension of $\mathcal{I}$, and thus on the Hausdorff dimension of $\mathcal{N}$, we use the following result (we refer the reader to [Fal03, Example 4.6] for its proof).

Lemma 2.12. Let $\mathcal{I}$ be a Cantor set constructed as above so that for all $l$, every interval $\mathcal{I}_{l-1}^{j}$ contains at least $\kappa_{l} \geq 2$ intervals $\mathcal{I}_{l}^{j^{\prime}}$, which are separated by gaps of at least $\epsilon_{l}$; assume furthermore that $\epsilon_{l}<\epsilon_{l-1}$. Then

$$
\operatorname{dim}_{\mathrm{H}} \mathcal{I} \geq \liminf _{l \rightarrow \infty} \frac{\log \left(\kappa_{1} \cdots \kappa_{l-1}\right)}{-\log \left(\kappa_{l} \epsilon_{l}\right)} .
$$

By our construction, the hypotheses of the above lemma hold with $\epsilon_{l}=$ $D^{\prime} \Lambda^{-\alpha n_{l}}$ and $\kappa_{l}=C^{\prime \prime} \Lambda^{-n_{l-1}+\alpha n_{l}}$. Let us then compute the liminf on the

\footnotetext{
3 That is, we do not consider endpoints of intervals $\mathcal{I}_{l-1}^{j}$
} 
right hand side of $(2)$; for any $l>0$ :

$$
\begin{aligned}
\log \left(\kappa_{1} \cdots \kappa_{l-1}\right)= & (l-1) \log C^{\prime \prime}+ \\
& +\left(\alpha n_{l-1}+(\alpha-1) n_{l-2}+\cdots+(\alpha-1) n_{1}-n_{0}\right) \log \Lambda \\
-\log \left(\kappa_{l} \epsilon_{l}\right)= & -\log \left(C^{\prime \prime} D^{\prime}\right)+n_{l-1} \log \Lambda .
\end{aligned}
$$

Given the super-exponential growth of $n_{l}$ we obtain:

$$
\liminf _{l \rightarrow \infty} \frac{\log \left(\kappa_{1} \cdots \kappa_{l-1}\right)}{-\log \left(\kappa_{l} \epsilon_{l}\right)}=\alpha=\frac{1}{2}-C / \log b .
$$

By Lemma 2.12 we can thus conclude that

$$
\operatorname{dim}_{\mathrm{H}} \mathcal{N} \geq \operatorname{dim}_{\mathrm{H}} \mathcal{I} \geq \frac{1}{2}-C / \log b,
$$

and, since $b$ can be chosen to be arbitrarily small, Proposition 1.4 (and thus the Main Theorem) is proved.

\section{Conclusions}

In this paper we obtained a lower bound on the Hausdorff dimension of the Newhouse parameter set for a family of smooth dissipative surface diffeomorphisms which nondegenerately unfolds a homoclinic tangency. In order to obtain our lower bound we take into account a specially designed class of non-simple sinks. It is natural to wonder about the optimal of our result: since we obtain this bound considering only a very special class of non-simple sinks, one would expect that the Hausdorff dimension of the Newhouse parameter set could indeed be larger than $1 / 2$.

In the same spirit, we should mention two works the Hausdorff dimension of related pathological phenomena:Lyu98 and [AM02. The first shows that the Hausdorff dimension of infinitely renormalizable parameters is at least $1 / 2$ (by focusing only on Misiurewicz renormalization), the second shows that this set has Hausdorff dimension less than 1. The latter shows also that the Hausdorff dimension of various other pathological phenomena (inexistence of physical measures, physical measures supported on expanding Cantor sets, non-ergodic physical measures) is positive.

Our understanding of the topological properties of the Newhouse set are still quite elusive and a more complete comprehension of the mechanisms of construction of higher complexity sinks could be the key to shed some more light on the subject.

We also believe that analogous results can be proved in the conservative setting; building from the renormalization techniques provided e.g. in MR97] and with constructions similar to the ones introduced in [DS13] and developed in this paper, we believe possible to show that the Hausdorff dimension of the Newhouse parameter set for a family of conservative diffeomorphisms which non-degenerately unfolds a homoclinic conservative is bounded below by $1 / 4$. 


\section{Appendix A. A modern proof of Newhouse Theorem 2.3}

Let us first recall the concept of Newhouse thickness, which will be instrumental in the proof of Theorem 2.3 .

Definition A.1. Given a Cantor set $K \subset \mathbb{R}$, a gap of $K$ is a connected component of $\mathbb{R} \backslash K$. Given a bounded gap $G$ of $K$ and $u$ in the boundary of $G$, a bridge $B$ of $K$ at $u$ is the connected component of $u$ in the complement of the union of the gaps longer than $|G|$. The thickness of $K$ at $u$ is: $\tau(K, u)=|B| /|G|$. The thickness of $K$, denoted by $\tau(K)$ is the infimum among these $\tau(K, u)$ for all boundary points $u$ of bounded gaps.

Definition A.2. If $K$ is a basic (hyperbolic) set of a $C^{2}$-diffeomorphism, the stable thickness of $K$, denoted by $\tau^{s}(K)$, is defined by

$$
\tau^{s}(K)=\limsup _{\epsilon \rightarrow 0} \tau\left(W_{\epsilon}^{u}(q) \cap W_{\eta}^{s}(K)\right),
$$

where $\eta>0$ and $q$ a point in $K$. Proposition 5 of [New79] states that this definition of thickness is independent of $\eta$ and $q \in K$. Thus it is well defined. The unstable thickness $\tau^{u}(K)$ is defined in a similar manner.

The following is the celebrated Newhouse Gap Lemma.

Lemma A.3 ([New79, Lemma 4], but see also [PT93, Section 4.2]). Let $K_{1}, K_{2} \subset \mathbb{R}$ be Cantor sets with thickness $\tau_{1}$ and $\tau_{2}$. If $\tau_{1} \cdot \tau_{2}>1$, then one of the three following possibilities occurs: $K_{1}$ is contained in a gap of $K_{2}$, $K_{2}$ is contained in a gap of $K_{1}, K_{1} \cap K_{2} \neq \emptyset$.

A.1. Hénon like maps close to Chebyshev quadratic polynomial. We now proceed to construct thick Cantor sets for Hénon-like maps in a range of parameters which bring them close to the (one-dimensional) dynamics of the Chebyshev quadratic polynomial. We proceed in two stages: in the first stage we construct thick Cantor sets for the one-dimensional dynamics; in the second one, we show that the hyperbolic continuation of such sets give thick invariant Cantor sets for the two-dimensional dynamics.

Let us first recall that any Hénon $C^{r}$-like family:

$$
f_{a}:(x, y) \mapsto\left(x^{2}+a+y,-b x\right)+\left(A_{a}(x, y), b B(x, y)\right)
$$

is $C^{r}$-close to the Hénon family $\left(h_{a, 0}\right)_{a}$ defined by $h_{a, 0}(x, y)=\left(x^{2}+y+a, 0\right)$. The restriction of $h_{a, 0}$ to the line $\mathbb{R} \times\{0\}$ is equal to $Q_{a}(x)=x^{2}+a$. The map $f_{a}$ is also $b$ - $C^{r}$-close to the real unimodal map $P(x)=x^{2}+a+A_{a}(x, 0)$. Up to conjugacy with a translation, we can assume that $D P(0)=0$. Since $P$ is unimodal, it has a two-branched inverse: one branch is orientation preserving and will be denoted with $P_{+}^{-1}$ and the other one is orientation reversing and will be denoted with $P_{-}^{-1}$; observe that, by construction, we have $P_{ \pm}^{-1}=\left(P \mid \mathbb{R}^{ \pm}\right)^{-1}$.

We will construct the wild basic set for $a$ close to -2 . For such $a, P$ has two fixed points: denote the orientation reversing fixed point by $\alpha \approx-1$ 
and the orientation preserving one by $\beta \approx 2$. We define inductively $\left(\alpha_{n}^{ \pm}\right)_{n \geq 0}$ as follows: let $\alpha_{0}^{-}=\alpha$ and $\alpha_{0}^{+}=P_{+}^{-1}(\alpha)$; then we let

$$
\alpha_{n}^{ \pm}:=P_{ \pm}^{-1}\left(\alpha_{n-1}^{+}\right) \in \mathbb{R}^{ \pm} .
$$

By linearizing the fixed point $\beta$ of $P$, we remark that $\left(\alpha_{n}^{+}\right)_{n}$ converges to $\beta$ and $\left(\alpha_{n}^{-}\right)_{n}$ converges to $P_{-}^{-1}(\beta)$. To fix notations, let $\alpha_{\infty}^{+}=\beta$ and $\alpha_{\infty}^{-}=$ $P_{-}^{-1}(\beta)$. Note that the distance between $\alpha_{n}^{ \pm}$and $\alpha_{\infty}^{ \pm}$is of the same order as $D P(\beta)^{-n}$. Define $\tilde{\alpha}_{1}^{ \pm}=\alpha_{0}^{ \pm}$; if the critical value $a$ of $P$ is smaller than $\alpha_{n-1}^{-}$, we define $\tilde{\alpha}_{n}^{ \pm}:=P_{ \pm}^{-1}\left(\alpha_{n-1}^{-}\right) \in \mathbb{R}^{ \pm}$. Likewise, if $a<\alpha_{\infty}^{-}$, define $\tilde{\alpha}_{\infty}^{ \pm}:=P_{ \pm}^{-1}\left(\alpha_{\infty}^{-}\right)$.

Two Cantor sets will be important for our purposes:

$$
\begin{aligned}
& C_{1}(P):=\bigcap_{k \geq 0} P^{-k}\left(\left[\alpha_{1}^{-}, \tilde{\alpha}_{2}^{-}\right] \sqcup\left[\tilde{\alpha}_{2}^{+}, \alpha_{1}^{+}\right]\right), \text {if } a<\alpha_{1}^{-} ; \\
& C_{2}(P):=\bigcap_{k \geq 0} P^{-k}\left(\left[\alpha_{\infty}^{-}, \tilde{\alpha}^{-} \infty\right] \sqcup\left[\tilde{\alpha}_{\infty}^{+}, \alpha_{\infty}^{+}\right]\right), \text {if } a<\alpha_{\infty}^{+} .
\end{aligned}
$$

For $r \geq 2, C_{1}(P)$ is a hyperbolic Cantor set, and we are going to show that $C_{2}(P)$ is also a hyperbolic Cantor set. Then we will compute the thickness of these sets. The hyperbolic continuation of $C_{1}(P)$ will be contained in the wild basic set $\tilde{K}$ for $f_{a}$, whereas the hyperbolic continuation of $C_{2}(\mathcal{R} P)$ will be embedded in $\tilde{K}$ as a basic set $K_{2}$, where $\mathcal{R} P$ is a pre-renormalization of $P$. A general picture of the construction is given Figure 1 .

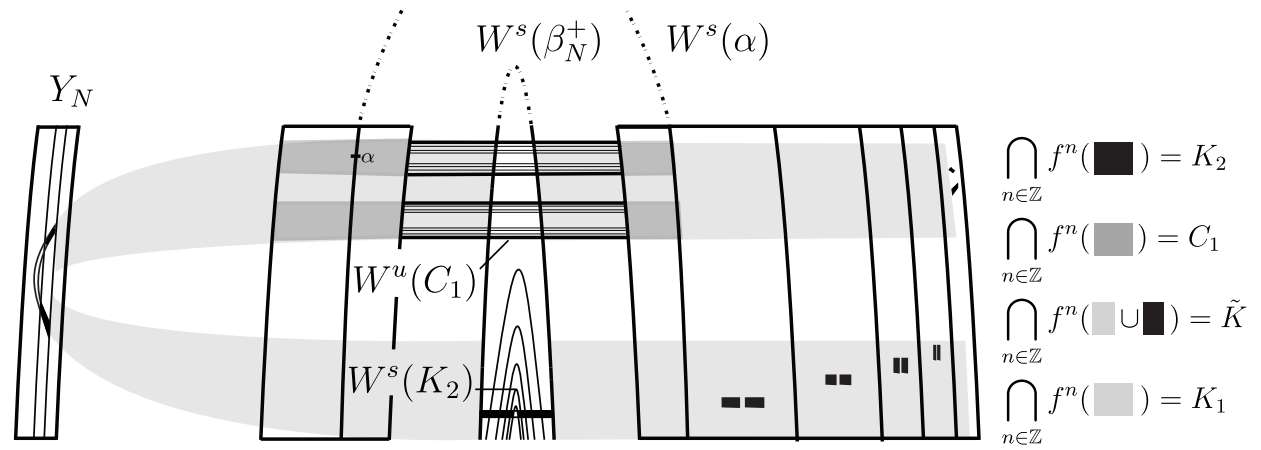

FiguRE 1. Sketch of the relative positions of the Cantor sets $C_{1}, K_{2}$ and $\tilde{K}$.

Let us now define $I_{0}=\tilde{I}_{0}=\left[\alpha_{0}^{-}, \alpha_{0}^{+}\right]$, and for $n \geq 1$ :

$$
I_{n}^{-}=\left[\alpha_{n}^{-}, \alpha_{n-1}^{-}\right] \quad I_{n}^{+}=\left[\alpha_{n-1}^{+}, \alpha_{n}^{+}\right] \quad \tilde{I}_{n}^{-}=\left[\tilde{\alpha}_{n-1}^{-}, \tilde{\alpha}_{n}^{-}\right] \quad \tilde{I}_{n}^{+}=\left[\tilde{\alpha}_{n}^{+}, \tilde{\alpha}_{n-1}^{+}\right]
$$

when well defined. Then they are diffeomorphically sent onto $I_{0}$ by $P^{n}$.

Lemma A.4. There exist $D>0$ and $\epsilon>0$, so that for every $P C^{2}-\epsilon$ close to $Q_{-2}$, whose critical value a satisfies $a<\alpha_{\infty}^{-}$, it holds that $C_{2}(P)$ is hyperbolic and its thickness is at least $D / \sqrt{\alpha_{\infty}^{-}-a}$. 
Proof. Let $\mathcal{A}:=\left\{\tilde{I}_{n}^{ \pm} ; n \geq 1\right\} \cup\left\{I_{0}\right\}$. Let $\mathcal{T}$ be formed by the pairs $\left(J, J^{\prime}\right) \in$ $\mathcal{A}^{2}$ so that $P(J) \supset J^{\prime}$ and $J \neq I_{0}$. A sequence of intervals $\left(J_{i}\right)_{i=1}^{n} \in \mathcal{A}^{n}$ is admissible if $\left(J_{i}, J_{i+1}\right)$ is in $\mathcal{T}$ for every $i$.

Sublemma A.5. There exists $C>0$ and $\epsilon>0$, so that for every $P C^{2}-\epsilon$ close to $Q_{-2}$ and satisfying $a<\alpha_{\infty}^{-}$, for every $N \geq 1$ and every admissible chain $\left(J_{i}\right)_{i=1}^{N} \in \mathcal{A}^{N}$, there exist diffeomorphisms $\phi$ and $\psi$ and an affine map A so that:

- the norm of $D A$ is at least $(1.5)^{N} / C$;

- $\phi \circ\left(P \mid J_{N}\right) \circ \cdots \circ\left(P \mid J_{2}\right) \circ\left(P \mid J_{1}\right) \circ \psi=A$;

- the $C^{2}$-norm of $\phi, \psi$ and their inverses are bounded by $C$.

This sublemma enables us to prove Lemma A.4 in fact, the central gap $G_{0} \ni 0$ of $C_{2}(P)$ has length of the order of $\sqrt{\alpha_{\infty}^{-}-a}$. For any other gap $G$ of $C_{2}(P)$, there exists an admissible chain $\left(J_{i}\right)_{i=1}^{N+1}$ ending at $J_{N+1}=I_{0}$, so that $G:=\left(P \mid J_{1}\right)^{-1} \circ\left(P \mid J_{2}\right)^{-1} \circ \cdots \circ\left(P \mid J_{N}\right)^{-1}\left(G_{0}\right)$. An associated bridge $B$ to $G$ has length at least the order of $\left(P \mid J_{1}\right)^{-1} \circ\left(P \mid J_{2}\right)^{-1} \circ \cdots \circ\left(P \mid J_{N}\right)^{-1}\left(B_{0}\right)$, where $B_{0}$ is a connected component of $I_{0} \backslash G_{0}$. Hence, by Sublemma A.5. the thickness associated to the gap $G$ is at least of the order of $\frac{\left|B_{0}\right|}{C^{2}\left|G_{0}\right|}$, which is of the same order as $1 / \sqrt{\alpha_{\infty}^{-}-a}$.

Proof of LemmaA.5. To prove this linearization, we only need to control the distortion of any admissible chain ending at $I_{0}$. It is well known that such a distortion is bounded if there exists $C>0$ and $\lambda>1.5$, so that for every admissible chain $\left(J_{i}\right)_{i=1}^{N+1}$ ending at $J_{N+1}=I_{0}$, the following hyperbolic times inequality holds:

$$
\left|D P^{N}(x)\right| \geq C \lambda^{N}, \forall x \in\left(P \mid J_{1}\right)^{-1} \circ\left(P \mid J_{2}\right)^{-1} \circ \cdots \circ\left(P \mid J_{N}\right)^{-1}\left(I_{0}\right) .
$$

Such an inequality is easy to prove when $J_{1}, \ldots, J_{N}$ is a sequence of the form $I_{N-1}^{ \pm}, I_{N-2}^{+}, \cdots, I_{1}^{+}, I_{0}$. To prove the general case, it is sufficient to show the existence of a bounded Riemannian metric $g$ on $I_{0}$ so that for every $x \in \tilde{I}_{n}^{ \pm}$, we have:

$$
\left|D P^{n}(x)\right|>(1.5)^{n} .
$$

The map $Q_{-2}(x)=x^{2}+a$ is expanding for the metric $g(x)=1 / \sqrt{x^{2}-4}$, which is bounded on $[-1,1]$. Hence for every $M \geq 0$, if $P$ is close enough to $Q_{-2}$, the restriction $P^{n} \mid \tilde{I}_{n}^{ \pm}$is expanding for the metric $g$ and every $M \geq n \geq$ $2\left(g \mid \cup_{n \leq M} I_{n} \cup \tilde{I}_{n}\right.$ is bounded). On the other hand, by using the linearization of the fixed point $\beta$, we remark that for $n$ sufficiently large, the derivative of $P^{n} \mid \tilde{I}_{n}^{ \pm}$is at least of the same order as $|D P(\beta)|^{n / 2}$.

Now that we have constructed invariant Cantor sets for the one-dimensional dynamics and that we have obtained bounds on their thicknesses, we proceed to consider the hyperbolic continuation of such invariant sets and estimate their thicknesses. 
Lemma A.6. For any $C^{2}$-Hénon like map $f$ close to $h_{-2,0}$, the Cantor set $C_{1}\left(Q_{-2}\right)$ persists as a basic set $C_{1}(f)$. The thickness $\tau^{u}\left(C_{1}(f)\right)$ is of the order of $b^{2}$, with $b:=\left|\operatorname{det} D_{0} f\right|$.

Similarly to Lemma A.4, we now prove:

Lemma A.7. There exist $C>0, \epsilon>0$, so that for every Hénon-like map

$$
f(x, y)=\left(x^{2}+y+a,-b x\right)+(A(x, y), b B(x, y)),
$$

such that $P(x):=x^{2}+a+A(x, 0)$ is $C^{2}-\epsilon$-close to $Q_{-2}$ and such that the critical value $a$ of $P$ satisfies $a<\alpha_{\infty}^{-}$and $b \ll\left(\alpha_{\infty}^{-}-a\right)$, then the hyperbolic continuation $C_{2}(f)$ of $C_{2}(P)$ is such that $\tau^{s}\left(C_{2}(f)\right) \geq C / \sqrt{\alpha_{\infty}^{-}-a}$.

Proof. The proof is the same as for Lemma A.4 provided that we show that the local unstable manifolds of $C_{2}(f)$ are $C^{2}$-close to horizontal lines. We now prove this horizontality condition.

Let $\beta(f)$ (resp. $\alpha(f)$ ) be the hyperbolic continuations of the orientation preserving (resp. reversing) fixed point $\beta$ (resp. $\alpha$ ) of $P$.

First we notice that local stable manifolds of $\beta(f)$ and $\alpha(f)$ are $b-C^{2}$-close to:

$$
\begin{aligned}
& W_{\mathrm{loc}}^{s}(\beta)=\{(x, y) \in \mathbb{R} \times[-1,+\infty): P(x)+y=\beta\} \\
& W_{\mathrm{loc}}^{s}(\alpha)=\{(x, y) \in \mathbb{R} \times[-1,+\infty): P(x)+y=\alpha\}
\end{aligned}
$$

By continuity, the hyperbolic continuation $C_{2}(f)$ of $C_{2}(P)$ must stay below $W_{\text {loc }}^{s}(\beta)$. Furthermore, from the analytic expression of $f, C_{2}(f)$ is included in the strip $b$-close to:

$$
\{(x, y) \in \mathbb{R} \times[-10 b, 10 b]: P(x)+y \leq \beta\}
$$

Recall that the central gap $G_{0}$ of $C_{2}(P)$ is given by $\left[\alpha_{\infty}^{-}, \alpha_{\infty}^{+}\right]$; similarly, hyperbolic continuations $\tilde{\alpha}_{\infty}^{ \pm}(f)$ of $\tilde{\alpha}_{\infty}^{ \pm}$have a local stable manifold which is $b-C^{2}$-close to:

$$
W_{\text {loc }}^{s}\left(\tilde{\alpha}_{\infty}^{ \pm}\right)=\left\{(x, y) \in \mathbb{R} \times[-1,+\infty): P(x)+y=\alpha_{\infty}^{-}\right\}
$$

It is a parabola with its top at $y$ of the order $\alpha_{\infty}^{-}-a$, and so large with respect to $b$. Hence this parabola cuts the strip $\mathbb{R} \times[-10 b, 10 b]$ at a distance of the order of $\sqrt{\alpha_{\infty}^{+}-a}$ of the line $x=0$.

By continuity the set $C_{2}(f)$ must be above this parabola. Hence $C_{2}(f)$ remains at a distance of the order of $\sqrt{\alpha_{\infty}^{-}-a}$ of the line $x=0$.

Thus at a neighborhood of $C_{2}(f)$, the cone

$$
\chi=\left\{(u, v) \in \mathbb{R}^{2}:|v| \leq|u| \sqrt{\alpha_{\infty}^{-}-a}\right\}
$$

is sent into itself, and so the local unstable manifolds are included in it. This implies that they are $C^{1}$-close to horizontal curves.

Let $Y_{e}$ be the intersection of $\mathbb{R} \times[-10 b, 10 b]$ with the domain below the local stable manifold of $\alpha(f)$ and above the local stable manifold of $\tilde{\alpha}_{\infty}^{ \pm}(f)$. We notice that $Y_{e}$ is close to the compact set $\left(\left[\alpha_{0}^{-}, \tilde{\alpha}_{\infty}^{-}\right] \cup\left[\tilde{\alpha}_{\infty}^{+}, \alpha_{0}^{+}\right]\right) \times\{0\}$. 
Similarly to the one dimensional case, every point $z \in C_{2}(f) \cap Y_{e}$ has infinitely many preimages in $Y_{e}$. From the same argument as for Lemma A.5, if $z^{\prime}$ is sent by $f^{n}$ to $z$, and $w \in \chi$, then:

$$
\left\|D_{z} f^{n}(w)\right\| \geq\left\|D_{z} f^{j}(w)\right\|, \quad \forall j \leq n .
$$

By [YW01, Lemma 2.4], we can conclude that the local unstable of $z$ has a curvature of the same order as $b$.

A.2. Misiurewicz pre-renormalization. In this subsection we quickly sketch the construction of the Misiurewicz pre-renormalization of a smooth family of Hénon-like maps; we refer once more the reader to Ber14 for more details.

Let, as before, $\left(f_{a}\right)_{a}$ be a $C^{2}$-Hénon like family of class $C^{4}$. Hence $f_{a}$ has the form $f_{a}(x, y)=\left(x^{2}+a+y,-b x\right)+\left(A_{a}(x, y), b B_{a}(x, y)\right)$. Put $P_{a}:=$ $x^{2}+a+A_{a}(x, 0)$.

The set $K\left(P_{a}\right):=\left\{\alpha_{n}^{ \pm}\left(P_{a}\right) ; n \geq 0\right\} \cup\left\{\alpha_{\infty}^{ \pm}\left(P_{a}\right)\right\}$ is hyperbolic for $P_{a}$. Let $K(f)$ be a hyperbolic continuation of $K\left(P_{a}\right) \times\{0\}$ for a map $f$. Hence for every continuation $k \in K(f)$ of $\left(x_{0}, 0\right) \in K\left(P_{a}\right) \times\{0\}$, a local stable manifold $W_{\mathrm{loc}}^{s}(k, f)$ of $k$ is $C^{2}$-close to the connected component of $\left(x_{0}, 0\right)$ in $\left\{(x, y) \in \mathbb{R} \times[-0.2,0.2]: x^{2}-2+y=x_{0}^{2}-2\right\}$.

For any $n \geq 0$, let $\hat{Y}_{n, f}^{ \pm}$be the rectangle bounded by the local stable manifolds $W^{s}(k, f)$ associated to the boundary points $k \in \partial I_{n}^{ \pm}$, and the lines $\left\{(x, y) \in \mathbb{R}^{2}: y= \pm 0.1\right\}$; let furthermore $\hat{I}_{n, f}^{ \pm}:=\hat{Y}_{k, f}^{ \pm} \cap\left\{(x, y) \in \mathbb{R}^{2}: y=0\right\}$.

Observe that by linearizing the fixed point $\beta^{+}$, there exists $C>0$, such that for every $0 \leq k \leq n$, we have:

$$
\forall x \in \hat{I}_{n, f}^{ \pm},\left|D P_{-2}^{k}(x)\right| \geq C\left|D P_{-2}\left(\beta^{+}\right)\right|^{k} .
$$

By Ber14, Section 2.2.2], there exists $\epsilon>0$, a $C^{4}$-neighborhood $V$ of the Hńon map $h_{-2,0}$, so that for each $f \in V$ which is $C^{2}$-Hénon like, any $n \geq 0$, there exist $C^{2}$-coordinates $y_{n, f}$ on an $\epsilon$-neighborhood $Y_{n, f}^{-}$of $\hat{I}_{n, f}^{-}$in $\hat{Y}_{n, f}^{-}$and $y_{n, f}^{\prime}$ on an $\epsilon$-neighborhood $Y_{0, f}$ of $\hat{I}_{0, f}$ in $\hat{Y}_{0, f}$ :

$$
y_{n, f}: \hat{I}_{n, f}^{-} \times[-\epsilon, \epsilon] \rightarrow Y_{n, f}^{-} \quad y_{n, f}^{\prime}: \hat{I}_{0, f} \times[-\epsilon, \epsilon] \rightarrow Y_{0, f} ;
$$

so that there exist $\sigma_{n, f}>3^{n}$ and $\lambda_{n, f}$ satisfying for $n \geq 6$ the following properties

- $y_{n, f}^{\prime-1} \circ f^{n} \circ y_{n, f}(x, y)=\left(\sigma_{n, f} \cdot x, \lambda_{n, f} \cdot y\right)$, for $(x, y) \in \hat{I}_{n, f}^{+} \times[-\epsilon, \epsilon]$;

- derivatives up to the second order of $(f, x, y) \mapsto y_{n, f}^{\prime}(x, y),(f, x, y) \mapsto$ $y_{n, f}(x, y)$ and their inverses $(f, x, y) \mapsto y_{n, f}^{-1}(x, y),(f, x, y) \mapsto y_{n, f}^{-1}(x, y)$ are bounded independently of $f \in V$ which is $C^{2}$-Hénon like (even if the determinant of $D f$ is zero!).

Since the unfolding of the homoclinic tangency is nondegenerate, for every $C^{2}$-Hénon like family $\left(f_{a}\right)_{a}$, for every $n \geq 2$, there exists $a_{n}$, with $a_{n} \approx-2$ 
when $n$ is large, so that for $f_{a} \in V, y_{n, f_{a}}^{-1} \circ f_{a} \circ y_{n, f_{a}}^{\prime}(x, y)$ is of the form:

$y_{n, f_{a}}^{-1} \circ f_{a} \circ y_{n, f_{a}}^{\prime}(x, y):(p+x, y) \mapsto\left(\xi x^{2}+\theta\left(a-a_{n}\right)+\gamma \cdot y, q+\zeta \cdot x\right)+E_{a}(x, y) \in \mathbb{R}^{2}$,

where $p \in \operatorname{int} \hat{I}_{0}, q \in(-\epsilon, \epsilon), \zeta$ are constants, and $\xi, \theta$ and $\gamma$ are non-zero constants (independent of $a) \sqrt[4]{4}$ and $E_{a}=\left(E_{a}^{1}, E_{a}^{2}\right) \in C^{2}\left(\mathbb{R} \times \mathbb{R}^{2}, \mathbb{R}^{2}\right)$ satisfies at $P:=(p, 0)$ :

$$
\left\{\begin{array}{l}
E_{a_{n}}^{1}(P)=\partial_{x} E_{a_{n}}^{1}(P)=\partial_{a} E_{a_{n}}^{1}(P)=\partial_{y} E_{a_{n}}^{1}(P)=\partial_{x x} E_{a_{n}}^{1}(P)=0 \\
E_{a_{n}}^{2}(P)=\partial_{x} E_{a_{n}}^{2}(P)=0 .
\end{array}\right.
$$

By [Ber14, Theorem 3.1], the family $\left(y_{n, f_{a}}^{\prime-1} \circ f_{a}^{n+1} \circ y_{n, f_{a}}^{\prime}\right)_{a}$ is conjugated to the Hénon $C^{2}$-like map $\mathcal{R}_{n} f_{a^{(n)}}$, with $\mathcal{R}_{n} f_{a^{(n)}(a)}=\Psi_{f_{a}} \circ y_{n, f_{a}}^{\prime-1} \circ f_{a}^{n+1} \circ$ $y_{n, f_{a}}^{\prime} \circ \Psi_{f_{a}}^{-1}$, where

$$
\begin{aligned}
\Psi_{f_{a}}(x, y) & :=\xi \cdot \sigma_{n, f_{a}} \cdot\left(x-p, \sigma_{n, f_{a}} \gamma y-\sigma_{n, f_{a}} \lambda_{n, f_{a}} q\right), \\
a^{(n)}(a) & :=\sigma_{n, f_{a}}^{2} \cdot\left(\xi \theta\left(a-a_{n}\right)+\lambda_{n, f_{a}} \xi \gamma q-\frac{\xi p}{\sigma_{n, f_{a}}}\right) .
\end{aligned}
$$

Moreover $\mathcal{R}_{n} f_{a^{(n)}(a)}$ is Hénon $C^{2}-\delta$-like with $\delta$ small when $n$ is large.

Remark A.8. If the determinant $b$ of $D f_{a}(0)$ is small, the determinant of $D \mathcal{R}_{n} f_{a^{(n)}(a)}$ is dominated by $b^{n+1}$ when $n$ is large.

A.3. Our proof of Theorem 2.3. We have now introduced all the results which we need in order to give the

Proof of Theorem 2.3. Let us consider a Hénon $C^{4}$-like family $\left(f_{a}\right)_{a}$ : recall that $f_{a}$ is of the form

$$
f_{a}:(x, y) \mapsto\left(x^{2}+a+y,-b x\right)+\left(A_{a}(x, y), b B_{a}(x, y)\right) .
$$

Define $P_{a}(x):=x^{2}+a+A_{a}(x, 0)$ and $\breve{f}_{a}(x, y):=\left(P_{a}(x)+y, 0\right)$. For $a<-1$, let $\beta_{a}^{+}$be the orientation preserving fixed point of $P_{a}$ and $\beta_{a}^{-}:=$ $\left(P_{a} \mid \mathbb{R}^{-}\right)^{-1}\left(\beta_{a}^{+}\right)$. We consider $a \approx-2$ such that $\beta_{a}^{-}-a$ is small and positive. For such values of $a$ we can define the Cantor set $C_{1}\left(P_{a}\right)$; the stable thickness of its hyperbolic continuation $C_{1}\left(f_{a}\right)$ is of order $b^{2}$ by Lemma A.6.

Also for many such values of $a$ we can perform the Misiurewicz prerenormalization $\mathcal{R}_{n} \breve{f}_{a^{(n)}}$ and $\mathcal{R}_{n} f_{a^{(n)}}$ which we have defined in the previous section, for large $n \geq 2$. Actually $\mathcal{R}_{n} \breve{f}_{a^{(n)}}$ preserves the horizontal line $\mathbb{R} \times\{0\}$ and its restriction is a unimodal map $\mathcal{R}_{n} P_{a^{(n)}}$. Let $\epsilon$ be the parameter appearing in Lemmata A.4 and A.7 then there exists $N_{\epsilon}$ so that for $N=N_{\epsilon}$ large enough and $a^{(N)}=a^{(N)}(a) \in[-3,0]$, this renormalization is a map $C^{2}$ - $\epsilon$-close to the quadratic map $x^{2}+a^{(N)}$. Note that the value of $N_{\epsilon}$ depends only on $\epsilon$ and the $C^{4}$-norm of $\left(f_{a}\right)_{a}$, as far as the family is $C^{2}$-Hénon like. We assume $a^{(N)}$ close to -2 . More precisely, if $\beta_{a^{(N)}}^{\prime+}$ denotes the orientation

\footnotetext{
${ }^{4}$ These constants $p, q, \zeta, \xi, \theta$ and $\gamma$ depend on $n$ and depend smoothly on the Hénon like family $\left(f_{a}\right)_{a}$.
} 
preserving fixed point of $\mathcal{R}_{N} P_{a^{(N)}}$ and $\beta_{a^{(N)}}^{--}:=\left(\mathcal{R}_{N} P_{a^{(N)}} \mid \mathbb{R}^{-}\right)^{-1}\left(\beta_{a^{(N)}}^{\prime+}\right)$, we assume $b$ and $a$ so that:

$$
0<\beta_{a^{(N)}}^{-}-a^{(N)}(a) \ll b^{4} .
$$

Hence by Lemma A.4, the Cantor set $C_{2}\left(\mathcal{R}_{N} P_{a^{(N)}}\right)$ has thickness large with respect to $b^{-2}$.

Also the same pre-renormalization of $\left(f_{a}\right)_{a}$ defines a map $\left(\mathcal{R}_{N} f_{a}\right)_{a}$ which is close to $\left(\mathcal{R}_{N} \breve{f}_{a}\right)_{a}$. By Remark A.8, the determinant $b_{N}$ of $D_{0} \mathcal{R}_{N} f_{a}$ is of the order of $b^{N+1}$, and so $\mathcal{R}_{N} f_{a}$ is $C^{2}-b^{N+1}$-close to $\mathcal{R}_{N} \breve{f}_{a}$ (for a large $N$ ). In order to apply Lemma A.7, we assume $b$ and $a$ so that at $a^{(N)}=a^{(N)}(a)$ :

$$
0<b^{n+1} \sim b_{N} \ll\left(\beta_{a^{(N)}}^{\prime-}-a^{(N)}\right)^{2} .
$$

Then, by Lemma A.7, there exist a constant $C$ and a hyperbolic horseshoe $C_{2}\left(\mathcal{R}_{N} f_{a^{(N)}}\right)$ of stable thickness greater than $C / \sqrt{\beta_{a^{(N)}}^{-}-a^{(N)}} \gg C / b^{2}$.

We remark that conditions $\left(\mathcal{C}_{1}\right)$ and $\left(\mathcal{C}_{2}\right)$ are simultaneously possible as far as $b^{8}$ is large with respect to $b^{N+1}$ (e.g. we shall assume $N \geq 8$ ). Moreover, for $N$ large but bounded, condition $\left(\mathcal{C}_{2}\right)$ remains possible for $b$ arbitrarily small.

The hyperbolic horseshoe $C_{2}\left(\mathcal{R}_{N} f_{a^{(N)}}\right)$ is embedded into the following basic set of $f_{a}$ :

$$
K_{2}\left(f_{a}\right):=\bigcup_{k=0}^{N-1} f_{a}^{k}\left(y_{N, a}^{\prime} \circ \Psi_{f_{a}}^{-1}\left(C_{2}\left(\mathcal{R}_{N} f_{a^{(N)}}\right)\right)\right),
$$

where $y_{N, a}^{\prime}$ and $\Psi_{f_{a}}^{-1}$ are the charts defining the Misiurewicz renormalization.

Since $C_{2}\left(\mathcal{R}_{N} f_{a^{(N)}}\right)$ is embedded into $K_{2}\left(f_{a}\right)$, we conclude that $K_{2}\left(f_{a}\right)$ has also stable thickness large with respect to $b^{2}$. Consequently,

$$
\tau^{u}\left(C_{1}\left(f_{a}\right)\right) \cdot \tau^{s}\left(K_{2}\left(f_{a}\right) \gg 1 .\right.
$$

We notice that, for some Riemannian metric, the expansion of $C_{1}\left(f_{a}\right)$ is close to 2 , whereas the expansion of $K_{2}\left(f_{a}\right)$ is close to $\sqrt[N]{2}$. This observation will be useful to prove condition $(i)$ of Theorem 2.3 .

Note that if we prove that there exist a basic set $\tilde{K}$ which contains both $C_{1}\left(f_{a}\right)$ and $K_{2}\left(f_{a}\right)$ and which has a homoclinic tangency, then by (3) and the Gap Lemma A.3 it is a wild hyperbolic set; this implies item (ii) of our statement. Let us first take care of constructing $\tilde{K}$ : the reader might find useful to refer to Figure 1 while reading through the description which follows.

We will use the following result, which is indeed just a rephrasing of [New79, Lemma 8]:

Lemma A.9. Assume there exist $z_{1}, z_{1}^{\prime} \in C_{1}\left(f_{a}\right)$ and $z_{2}, z_{2}^{\prime} \in K_{2}\left(f_{a}\right)$, local unstable manifolds $W_{\mathrm{loc}}^{u}\left(z_{1}\right), W_{\mathrm{loc}}^{u}\left(z_{2}\right)$ and local stable manifolds $W_{\mathrm{loc}}^{s}\left(z_{1}^{\prime}\right)$, $W_{\mathrm{loc}}^{s}\left(z_{2}^{\prime}\right)$ such that $W_{\mathrm{loc}}^{u}\left(z_{1}\right)$ intersects $W_{\mathrm{loc}}^{s}\left(z_{2}^{\prime}\right)$ transversely at a point $w$, 
$W_{\text {loc }}^{u}\left(z_{2}\right)$ intersects $W_{\text {loc }}^{s}\left(z_{1}^{\prime}\right)$ transversely at a point $w^{\prime}$, and $w, w^{\prime} \notin C_{1}\left(f_{a}\right) \cup$ $K_{2}\left(f_{a}\right)$. Then there exists a basic set $\tilde{K}_{a}$ containing both $C_{1}\left(f_{a}\right)$ and $K_{2}\left(f_{a}\right)$.

Moreover, in order to prove condition $(i)$ of Theorem 2.3, we shall also prove that the intersection points $w, w^{\prime}$ enjoy an expansion which is uniformly bounded from below when $b$ is small.

To this end, let us go back to the one dimensional dynamics, which appears at the limit when $b \rightarrow 0$. More precisely, when $b$ approaches 0 , Conditions $\left(\mathcal{C}_{1}\right)$ and $\left(\mathcal{C}_{2}\right)$ imply $a$ to be such that $a \in I_{N}$, and $P_{a}^{N+1} \mid P_{a}^{-1}\left(I_{N}\right)$ is conjugated to the Chebyshev map (both restricted to their maximal invariant compact set).

Let $\beta_{N}^{+}$be the orientation preserving fixed point of $P_{a}^{N+1} \mid P^{-1}\left(I_{N}\right)$. Let $\beta_{N}^{-}$be the preimage of $\beta_{N}^{+}$by $P_{a}^{N+1} \mid P_{a}^{-1}\left(I_{N}\right)$. From classical unimodal theory, the maximal invariant set:

$$
K_{1}\left(P_{a}\right):=\bigcap_{n \geq 0} P_{a}^{-n}\left(\left[\beta_{a}^{-}, \beta_{a}^{+}\right] \backslash\left(\beta_{N}^{+}, \beta_{N}^{-}\right)\right)
$$

is hyperbolic (one can even show that its expansion is at least of the order of $\sqrt[N]{2})$ and transitive. This basic set persists as a basic set $K_{1}\left(f_{a}\right)$ for $b$ small, with a similar expansion.

We notice that $K_{1}\left(f_{a}\right) \cap K_{2}\left(f_{a}\right)$ contains the hyperbolic continuation $\beta_{N}^{+}\left(f_{a}\right)$ of $\beta_{N}^{+}$. Since both $K_{1}\left(f_{a}\right)$ and $K_{2}\left(f_{a}\right)$ are hyperbolic Cantor sets, we can find $z_{1}, z_{1}^{\prime} \in K_{1}\left(f_{a}\right)$ and $z_{2}, z_{2}^{\prime} \in K_{2}\left(f_{a}\right)$ all close to $\beta_{N}^{+}\left(f_{a}\right)$ such that $W_{\text {loc }}^{u}\left(z_{1}\right)$ intersects $W_{\text {loc }}^{s}\left(z_{2}^{\prime}\right)$ transversely at a point $w, W_{\text {loc }}^{u}\left(z_{2}\right)$ intersects $W_{\text {loc }}^{s}\left(z_{1}^{\prime}\right)$ transversely at a point $w^{\prime}$, and $w, w^{\prime} \notin K_{1}\left(f_{a}\right) \cup K_{2}\left(f_{a}\right)$. Hence, using Lemma A.9 we prove the existence of a basic set $\tilde{K}_{a}$ containing both $K_{1}\left(f_{a}\right) \supset C_{1}\left(f_{a}\right)$ and $K_{2}\left(f_{a}\right)$, with expansion bounded from below uniformly on $b$ (actually at least of the order of $\sqrt[N]{2}$ ).

In order to conclude the proof, we now need to show that the basic set $\tilde{K}_{a}$ has a homoclinic tangency for some $a$ which satisfies $\left(\mathcal{C}_{1}\right)$ and $\left(\mathcal{C}_{2}\right)$; to this end observe that the local stable manifold $W_{\text {loc }}^{s}\left(\beta_{N}^{+}\left(f_{a}\right)\right)$ is $b-C^{2}$-close to the parabola

$$
\left\{(x, y) \in[-1,+\infty): x^{2}+y=\beta_{N}^{+}\right\}
$$

Since $K_{1}\left(f_{a}\right)$ contains $\beta_{N}^{+}\left(f_{a}\right)$, the local stable manifold $W_{\mathrm{loc}}^{s}\left(\beta_{N}^{+}\left(f_{a}\right)\right)$ is $C^{2}$ accumulated by other local stable manifolds $\left(W_{n}^{s}(a)\right)_{n}$ of points in $K_{1}\left(f_{a}\right)$; the parabolas $W_{n}^{s}$ are above $W_{\text {loc }}^{s}\left(\beta_{N}^{+}\left(f_{a}\right)\right)=: W_{\infty}^{s}(a)$.

If $\sigma$ denotes the unstable expansion of $D f^{N+1}\left(\beta_{N}^{+}\left(f_{a}\right)\right)$, there exists $C>0$ independent of $\left(f_{a}\right)_{a}$ (and so of $b$ ), but depending on $N$ already fixed, such that we can assume, for any $n$ :

$$
\sigma^{-n} / C<d\left(W_{n}^{s}(a), W_{\infty}^{s}(a)\right)<C \sigma^{-n} .
$$

By taking $C$ larger, we can examine the images of $\left(W_{n}^{s}\right)_{n \in\{0, \ldots, \infty\}}$ by the renormalization chart $\phi_{a}:=\Psi_{f_{a}} \circ y_{N, f_{a}}^{\prime-1}$ and obtain, for any $n$ :

$$
\sigma^{-n} / C<d\left(\phi_{a}\left(W_{n}^{s}\right), \phi_{a}\left(W_{\infty}^{s}(a)\right)\right)<C \sigma^{-n}
$$


Furthermore, the parameter dependence of $\left(\phi_{a}\left(W_{n}^{s}\right)\right)_{n \in\{0, \ldots, \infty\}}$ depends continuously on $n$ in the one point compactification $\{0, \ldots, \infty\}$ of $\mathbb{N}$.

On the other hand, a certain local unstable manifold $W^{u}$ of $\phi_{a}\left(K_{2}\left(f_{a}\right)\right)$ is $b^{N+1}-C^{2}$-close to the curve

$$
\left\{\mathcal{R}_{N} f_{a^{(N)}(a)}(t, 0): t \in[-1,1]\right\} .
$$

This curve has a fold which is $b^{N+1}$-close to $\left(a^{(N)}(a), 0\right)$. Also, there exists $a_{\infty}^{(N)}$ which is $b^{N+1}$-close to $\beta_{a_{\infty}^{(N)}}^{(-}$so that $W^{u}\left(a_{\infty}^{(N)}\right)$ is tangent to the local stable manifold $\phi_{a}\left(W_{\infty}^{s}\left(a_{\infty}^{(N)}\right)\right)$, and the unfolding of this tangency is nondegenerate. So there are parameters $a_{n}$ so that $W^{u}\left(a^{(N)}\left(a_{n}\right)\right)$ is tangent to the local stable manifold $\phi_{a}\left(W_{\infty}^{s}\left(a^{(N)}\left(a_{n}\right)\right)\right)$ and satisfy (by taking $C$ larger) for any $n$ :

$$
\sigma^{-n} / C<d\left(a_{n}^{(N)}, a_{\infty}^{(N)}\right)<C \sigma^{-n} .
$$

Consequently there exists $n$ so that $a^{(N)}\left(a_{n}\right)$ satisfies $\left(\mathcal{C}_{1}\right)$ and $\left(\mathcal{C}_{2}\right)$.

\section{REFERENCES}

[AM02] A. Avila and C. G. Moreira. Hausdorff dimension and the quadratic family. Manuscript, 2002.

[Ber14] Pierre Berger. Normal forms and Misiurewicz renormalization for dissipative surface diffeomorphisms. ArXiv e-prints, 2014.

[Bow70] Rufus Bowen. Markov partitions for Axiom A diffeomorphisms. Amer. J. Math., 92:725-747, 1970.

[CP10] S. Crovisier and E. R. Pujals. Essential hyperbolicity and homoclinic bifurcations: a dichotomy phenomenon/mechanism for diffeomorphisms. ArXiv e-prints, November 2010.

[DL13] R. Dujardin and M. Lyubich. Stability and bifurcations for dissipative polynomial automorphisms of $\mathrm{C}^{\wedge} 2$. ArXiv e-prints, May 2013.

[DS13] Jacopo De Simoi. On cyclicity-one elliptic islands of the standard map. J. Mod. Dyn., 7(2):153-208, 2013.

[Dua99] Pedro Duarte. Abundance of elliptic isles at conservative bifurcations. Dynam. Stability Systems, 14(4):339-356, 1999.

[Dua08] P. Duarte. Elliptic isles in families of area-preserving maps. Ergodic Theory Dynam. Systems, 28(6):1781-1813, 2008.

[Fal03] Kenneth Falconer. Fractal geometry. John Wiley \& Sons Inc., Hoboken, NJ, second edition, 2003. Mathematical foundations and applications.

[GK07] A. Gorodetski and V. Kaloshin. How often surface diffeomorphisms have infinitely many sinks and hyperbolicity of periodic points near a homoclinic tangency. Adv. Math., 208(2):710-797, 2007.

[GS03] S. V. Gonchenko and L. P. Shil'nikov. On two-dimensional area-preserving maps with homoclinic tangencies that have infinitely many generic elliptic periodic points. Zap. Nauchn. Sem. S.-Peterburg. Otdel. Mat. Inst. Steklov. (POMI), 300(Teor. Predst. Din. Sist. Spets. Vyp. 8):155-166, 288-289, 2003.

[HPS77] M. W. Hirsch, C. C. Pugh, and M. Shub. Invariant manifolds. Springer-Verlag, Berlin, 1977. Lecture Notes in Mathematics, Vol. 583.

[Lyu98] Mikhail Lyubich. How big is the set of infinitely renormalizable quadratics? In Voronezh Winter Mathematical Schools, volume 184 of Amer. Math. Soc. Transl. Ser. 2, pages 131-143. Amer. Math. Soc., Providence, RI, 1998. 
[MR97] Leonardo Mora and Neptalí Romero. Moser's invariant curves and homoclinic bifurcations. Dynam. Systems Appl., 6(1):29-41, 1997.

[MSS83] R. Mañé, P. Sad, and D. Sullivan. On the dynamics of rational maps. Ann. Sci. École Norm. Sup. (4), 16(2):193-217, 1983.

[New74] Sheldon. E. Newhouse. Diffeomorphisms with infinitely many sinks. Topology, 12:9-18, 1974.

[New79] Sheldon E. Newhouse. The abundance of wild hyperbolic sets and nonsmooth stable sets for diffeomorphisms. Inst. Hautes Études Sci. Publ. Math., (50):101151, 1979.

[Pal08] J. Palis. Open questions leading to a global perspective in dynamics. Nonlinearity, 21(4):T37-T43, 2008.

[PS00] Enrique R. Pujals and Martín Sambarino. Homoclinic tangencies and hyperbolicity for surface diffeomorphisms. Ann. of Math. (2), 151(3):961-1023, 2000.

[PT93] Jacob Palis and Floris Takens. Hyperbolicity and sensitive chaotic dynamics at homoclinic bifurcations, volume 35 of Cambridge Studies in Advanced Mathematics. Cambridge University Press, Cambridge, 1993. Fractal dimensions and infinitely many attractors.

[Rob83] Clark Robinson. Bifurcation to infinitely many sinks. Comm. Math. Phys., 90(3):433-459, 1983.

[Sel85] George R. Sell. Smooth linearization near a fixed point. Amer. J. Math., 107(5):1035-1091, 1985.

[TLY86] Laura Tedeschini-Lalli and James A. Yorke. How often do simple dynamical processes have infinitely many coexisting sinks? Comm. Math. Phys., 106(4):635$657,1986$.

[Wan90] Xiao-Jing Wang. The Newhouse set has a positive Hausdorff dimension. Comm. Math. Phys., 131(2):317-332, 1990.

[Yoc95] Jean-Christophe Yoccoz. Introduction to hyperbolic dynamics. In Real and complex dynamical systems (Hillerød, 1993), volume 464 of NATO Adv. Sci. Inst. Ser. C Math. Phys. Sci., pages 265-291. Kluwer Acad. Publ., Dordrecht, 1995.

[YW01] L. S. Young and Q. D. Wang. attractors with one direction of instability. Commun. Math. Phys., 218:1-97, 2001.

Pierre Berger, Laboratoire Analyse, Géométrie \& Applications CNRS-UMR 7539 Institut Galilée Université Paris 13, 99 avenue J.B. Clément - 93430 VilLETANEUSE - FRANCE

E-mail address: pierre.berger@math.univ-paris13.fr

$U R L:$ http://www . math. sunysb.edu/ berger/

Jacopo De Simoi, Department of Mathematics, University of Toronto, 40

St George St. Toronto, ON, Canada M5S 2E4

E-mail address: jacopods@math.utoronto.ca

$U R L:$ http://www.math.utoronto.ca/jacopods 